\title{
An experimental method to determine the electrostatic field enhancement factor of a practical conductor surface
}

\section{McAllister, lain Wilson; Crichton, George C}

Published in:

IEEE Transactions on Electrical Insulation

Link to article, DOI:

$10.1109 / 14.90290$

Publication date:

1989

Document Version

Publisher's PDF, also known as Version of record

Link back to DTU Orbit

Citation (APA):

McAllister, I. W., \& Crichton, G. C. (1989). An experimental method to determine the electrostatic field

enhancement factor of a practical conductor surface. IEEE Transactions on Electrical Insulation, 24(2), 325-333. https://doi.org/10.1109/14.90290

\section{General rights}

Copyright and moral rights for the publications made accessible in the public portal are retained by the authors and/or other copyright owners and it is a condition of accessing publications that users recognise and abide by the legal requirements associated with these rights.

- Users may download and print one copy of any publication from the public portal for the purpose of private study or research.

- You may not further distribute the material or use it for any profit-making activity or commercial gain

- You may freely distribute the URL identifying the publication in the public portal 


\title{
An Experimental Method to Determine the Electrostatic Field Enhancement Factor of a Practical Conductor Surface
}

\author{
I. W. McAllister and G. C. Crichton \\ Physics Laboratory II, \\ The Technical University of Denmark, Lyngby, \\ Denmark
}

\begin{abstract}
A method of determining the field enhancement factor of a practical conductor is presented. The method is developed from a modified theory of discharge onset in a gaseous medium. This modification incorporates the influence of conductor surface roughness. Thereafter onset data from an experimental study which utilized electrodes of varying surface roughness are examined, and the results obtained using the proposed method are discussed with reference to both the underlying theory and the practical aspects of the experimental measurements.
\end{abstract}

\section{INTRODUCTION}

$\mathrm{F}$ ollowiNg the introduction of compressed $\mathrm{SF}_{6}$ as an insulating medium, electrode surface roughness was found to cause a pronounced reduction in the anticipated insulation level of a system. This apparent reduction in the dielectric strength of compressed $\mathrm{SF}_{6}$ can be accounted for by considering the electrostatic fields of rough surfaces $[1,2]$.

In practice, all conductors exhibit a rough surface, with the degree of roughness being dependent upon the production process employed $[3,4]$. This inherent microscopic surface roughness leads to a perturbation of the macroscopic electric field such that the maximum field strength $E_{\max }$ of a rough surface will be greater than the maximum field strength $E_{a}$ of the associated (idealized) macroscopic geometry. This increase in the field strength due to a rough surface can be quantified in terms of a field enhancement factor $m$, where $m$ is defined as

$$
m \equiv \frac{E_{\max }}{E_{a}}
$$

with $m \geq 1$.

The influence of different geometric parameters upon $m$ can be assessed by examining roughness models which incorporate simple protrusion geometries. However, as the microscopic geometry of practical surfaces is extremely complex, analytical studies cannot provide the actual $m$ values associated with such surfaces.

In the present paper, we circumvent this limitation by proposing an experimental determination of the intrinsic $m$ value of a rough surface. The method is based

0018-9367/89/0400-325\$1.00 (C) 1889 IEEE 
upon the detection of discharge onset in a gaseous dielectric at the surface in question. The associated onset voltage levels, which are recorded for a range of gas pressures, are analyzed to yield $m$. Results obtained by use of the method are presented and the limitations in the technique are discussed.

\section{SURFACE ROUGHNESS FACTOR $\xi$}

$\mathrm{T}$ HIS factor expresses the reduction in the insulation 1 strength of a compressed gas system from that predicted theoretically on the basis of the streamer criterion applied to the idealized macroscopic geometry [5]. Owing to the geometrical complexity of the microscopic structure of conductor surfaces, the value of this parameter can only be determined experimentally. However, an appreciation of the behavior of $\xi$ can be obtained by considering simple models of a rough surface $[1,2,4,6,7]$.

For a hemispherical protrusion, Pedersen $[1,2]$ derived the variation of $\xi$ for $\mathrm{SF}_{6}$, see Figure 1. This diagram indicates that as $p / H_{i} \rightarrow \infty, \xi$ tends asymptotically to some limiting value, $\xi_{\text {lim }}$, where $p$ is the gas pressure and $H_{i}$ is the mean curvature of the protrusion. In this case, $H_{i}=1 / R$ where $R$ is the protrusion radius. The value of $\xi_{\text {lim }}$ can be deduced as follows.

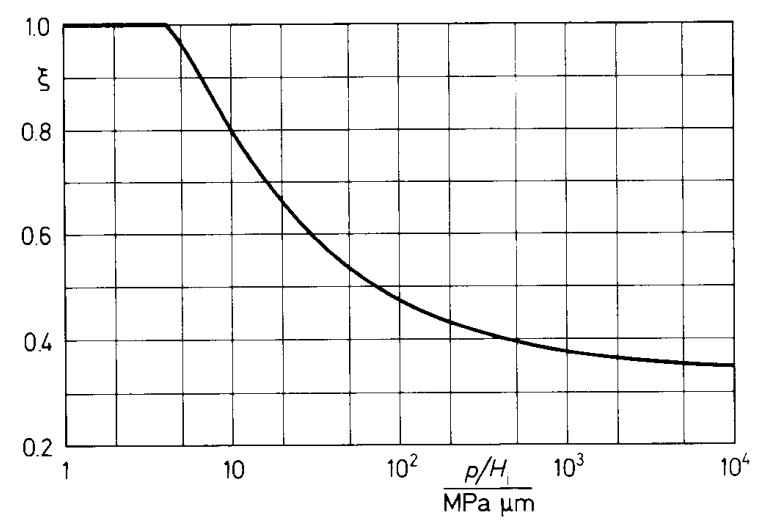

Figure 1.

Surface roughness factor $\xi$ for $\mathrm{SF}_{6}[2]$.

For (ideal) macroscopic geometries it is known $[1,2]$ that, for increasing gas pressure, the value of the theoretical onset field strength $E_{a t}$ tends to $E_{l i m}$, where $E_{l i m}$ is the limiting field strength for ionization processes; see also (11) and (12). In a corresponding manner the onset field strength for a rough surface, which is the appropriate value of $E_{\max }$, will likewise tend to $E_{l i m}$ at high gas pressures. (In effect the value of $p / H_{i}$ becomes a macroscopic quantity at large $p$ ).

In analytical studies of the influence of surface roughness, $\xi$ is defined in terms of the macroscopic field strength pertaining at onset $E_{a}$ and the theoretically predicted onset value $E_{a t} ; v i z$.

$$
\xi \equiv \frac{E_{a}}{E_{a t}}
$$

with $\xi \leq 1$. Taken together with (1), this leads to

$$
\xi=\frac{E_{\text {max }}}{m E_{a t}}
$$

Consequently as $E_{m a x} \rightarrow E_{l i m}$ and $E_{a t} \rightarrow E_{l i m}$ as $p \rightarrow \infty$, we will have $\xi \rightarrow 1 / m$, and in the limit

$$
\xi_{\text {lim }}=\frac{1}{m}
$$

Confirmation of this result can be obtained from specific models. For example, from the mathematical expressions given in $[1,2]$ for a hemispherical protrusion $(m=3)$, we can express $\xi$ explicitly as

$$
\xi=\frac{2}{3}\left[1-\sin \left(\frac{\theta}{3}\right)\right]
$$

with

$$
\theta=\arcsin \left[2\left(1-\frac{H_{i} M}{p}\right)^{3}-1\right]
$$

The parameter $M$ is the figure of merit for a strongly electronegative gas [8]. From (6), it is evident that, as $p / H_{i} \rightarrow \infty, \theta \rightarrow \frac{\pi}{2}$ and thus $\xi \rightarrow \frac{1}{3}$, in agreement with the relevant $m$ value; see also Figure 1 .

\section{INFLUENCE OF PROTRUSION CURVATURE $H_{i}$}

For a regular point of a surface, the mean curvature $H$ is given by

$$
2 H=\frac{1}{\rho_{1}}+\frac{1}{\rho_{2}}
$$

where $\rho_{1}$ and $\rho_{2}$ are the principal radii of curvature. As the tip of any protrusion will invariably form a convex surface, this implies that the radii of curvature have the same sign and consequently the values of $\rho_{1}$ and $\rho_{2}$ must fall into one of three categories: 
1. $\rho_{1}$ finite, $\rho_{2}$ finite and $\rho_{1}=\rho_{2}$ : axial symmetry.

2. $\rho_{1}$ finite, $\rho_{2}$ finite and $\rho_{1} \neq \rho_{2}$ : no simple symmetry. 3. $\rho_{1}$ finite, $\rho_{2}$ infinite: cylindrical symmetry.

Using different protrusion models, a study of the effect of this variation in $\rho_{1}$ and $\rho_{2}$ upon $\xi$ has been undertaken. Although full details of this study will be published at a later date, an essential result for the present investigation is shown in Figure 2. This diagram illustrates, for categories (1) and (3), the variation of $\xi$ for a constant $m$. It is clear that, as $p /\left(H_{i} M\right) \rightarrow \infty, \xi \rightarrow 1 / m$ irrespective of the $\rho_{1}, \rho_{2}$ variation. This behavior implies that with a knowledge of the $\xi$ variation for any rough surface the specific $m$ value associated with that surface can, in theory, be determined.

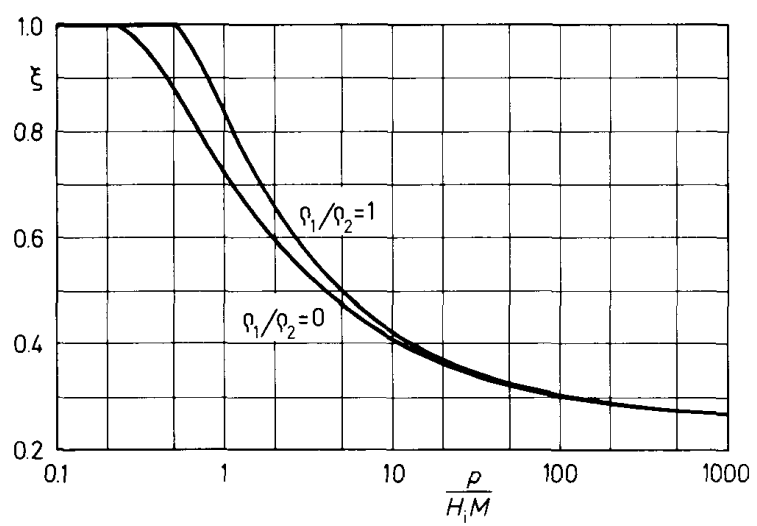

Figure 2.

Influence of electrode microscopic curvature $\boldsymbol{H}_{i}$ upon the surface roughness factor $\xi$.

\section{DETERMINATION OF $\xi_{\text {lim }}$}

\section{THEORETICAL BACKGROUND}

On the basis of the streamer criterion [8], the discharge onset voltage $U_{0}$ for any electrode geometry may be expressed as a product of the field utilization factor $\eta$, the surface curvature factor $\zeta$, the surface roughness factor $\xi$, the gas pressure $p$, the gap length $d$ and the limiting $E / p$ of the gaseous dielectric; viz.

$$
U_{o}=n \eta \xi\left(\frac{E}{p}\right)_{l i m} p d
$$

where $(E / p)_{\text {lim }}$ is the value of $(E / p)$ for which the value of the effective coefficient of ionization $\bar{\alpha}$ is zero. From (8) we have

$$
\xi=\frac{U_{o}}{\eta \zeta\left(\frac{E}{p}\right)_{l i m} p d}
$$

Of the parameters on the RHS of (9), $U_{0}$ may be determined experimentally for selected values of $p$ and $d$, while the $(E / p)_{l i m}$ of the gaseous dielectric is assumed to be known.

The utilization factor $\eta$ is a function of the macroscopic gap geometry and potential boundary conditions alone, and is defined as

$$
\eta \equiv \frac{U}{E_{a} d}
$$

with $E_{a}$ being the maximum field strength of the idealized macroscopic geometry associated with a potential difference $U$. Hence $\eta$ can be evaluated by any field calculating method. $\zeta$, the surface curvature factor, is associated with the idealized macroscopic conductor geometry and is defined as

$$
\zeta \equiv \frac{E_{a t}}{E_{l i m}}
$$

with $\zeta \geq 1 . \quad E_{a t}$ is the theoretically predicted onset field strength for the idealized macroscopic geometry. For practical purposes, the surface curvature factor for a strongly electronegative gas can be expressed $[2,7]$ as

$$
\zeta=1+\sqrt{\frac{4 H_{a} M}{p}}
$$

where $H_{a}$ represents the mean curvature of the idealized macroscopic conductor geometry. Consequently, as both $\eta$ and $\zeta$ can be evaluated from a knowledge of the gap geometry and the test gas parameters, measurements of $U_{o}$ will provide $\xi$ data.

When deriving the onset conditions in analytical studies, the mathematics leads naturally to $H_{i} M / p$ being expressed as a function of $\xi$. In general it is not possible to invert such relationships and express $\xi$ as a function of $H_{i} M / p$. This situation applies to the $\xi$ 
variations shown in Figure 2. However, an examination of (5) suggests that $\xi$ expression for such curves can be obtained. For $H_{i} M / p<1$, the sine term in (5) can be expanded as a power series in $H_{i} M / p$ to give an expression of the form

$$
\xi=a_{0}+a_{1}\left(\frac{H_{i} M}{p}\right)+a_{2}\left(\frac{H_{i} M}{p}\right)^{2}+a_{3}\left(\frac{H_{i} M}{p}\right)^{3}+\ldots
$$

with in this instance $a_{0}=1 / 3$, i.e., as $H_{i} M / p \rightarrow 0$, $\xi \rightarrow a_{0}=\xi_{\text {lim }}$. Consequently, on the basis of (13), the determination of $\xi_{\text {lim }}$ and hence $m$ should be feasible from a regression analysis of the experimental $\xi$ data.

\section{PRACTICAL ASPECTS}

Up to this point, the background theory on the determination of $m$ has been developed in relation to protrusion models of known geometry, such that $\xi$ is a function of $\left(H_{i} M / p\right)$. In practice however, the geometry of rough surfaces is so ill-defined that a knowledge of $H_{i}$ will not be available, to which we must add the uncertainty in the numerical values of $M$ to be employed at the higher gas pressures. As the influence of surface roughness becomes significant at $p>0.1 \mathrm{MPa}$, the $M$ data should be available over the appropriate pressure range. At present, the $M$ data for different gases are restricted to low values of gas pressure, $p \leq 0.1 \mathrm{MPa}$ [9-12], and although extrapolation is possible the uncertainty may be increased further due to the non-ideal behavior of many strongly electronegative gases [13]. This uncertainty in $M$ is fortunately but a negligible limitation with reference to the $\zeta$ evaluation since, for the values of $H_{a}$ and $p$ encountered in practice, the square root term in $(12)$ is $\ll 1$.

Because $H_{i}$ is inherently unknown, this parameter presents a major quantitative difficulty in the $\xi_{\text {lim }}$ evaluation. Nevertheless, the lack of appropriate values for both $H_{i}$ and $M$ can be elegantly circumvented by analyzing the variation of $\xi$ in terms of $p$ alone, and not the dimensionless parameter $\left(H_{i} M / p\right)$ as the theory indicates; the factor $H_{i} M$ is simply included in the regression coefficients.

\section{EXPERIMENTAL RESULTS}

T $N$ a parallel study of the insulating characteristics 1 of different strongly electronegative gases, discharge onset voltages for $\mathrm{SF}_{6}$ are reported $[14,15]$. These measurements, which were obtained for a rough surface environment, will now be analyzed in the light of the present theory. For completeness, a brief description of the pertinent experimental aspects is provided.

The $\mathrm{SF}_{6}$ discharge measurements were undertaken with a coaxial cylinder gap over the pressure range $0.05<$ $p<0.9 \mathrm{MPa}$. The inner electrode had a nominal radius of $11.0 \mathrm{~mm}$ while the gap distance was $19.0 \mathrm{~mm}$. The length of the constant diameter section of the outer electrode was $80 \mathrm{~mm}$ whereas that of the inner was $450 \mathrm{~mm}$. The overall length of the flared outer electrode was 160 $\mathrm{mm}$.

Inner electrodes with different surface roughness magnitudes were employed. The surface finish of these electrodes was produced by a turning process and thereafter the respective $R_{a}$ values [3] were determined. In this parallel study, $U_{o}$ measurements were undertaken with $R_{a}$ values of 5,20 , and $35 \mu \mathrm{m}$.

Discharge onset (corona or direct breakdown) was detected oscillographically for a negative dc applied voltage. No artificial irradiation was used and the applied voltage was raised at $\sim 1 \mathrm{kV} / \mathrm{min}$. In the absence of direct breakdown, discharge onset was identified with the initial appearance of pre-breakdown current pulses, see $[16,17]$.

The measured onset voltage levels for the three values of $R_{a}$ are presented in Figure 3 as a function of gas pressure. For comparison, the calculated onset voltage characteristic for the idealized macroscopic geometry $\left(R_{a}=0\right)$ is included. This latter curve is derived using (8) with $\xi=1$ [1]. As indicated previously, $\xi$ is the ratio of the actual onset voltage to the corresponding theoretical value and thus the results in Figure 3 can be used to determine $\xi$.

As seen from Figure 3, the $U_{o}$ measurements were performed at pressures exceeding $0.1 \mathrm{MPa}$, and thus the non-ideal gas behavior of $\mathrm{SF}_{6}[18]$ must be taken into 


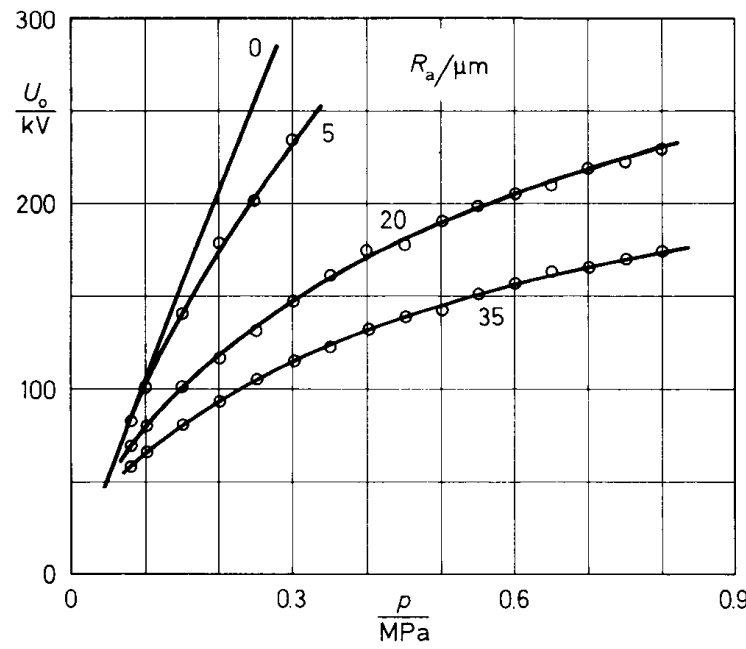

Figure 3.

Discharge onset voltage $U_{o}$ in $\mathrm{SF}_{6}$ as a function of gas pressure $p$ for different $R_{a}$ values.

account in the $\xi$ evaluation. In such circumstances the ideal gas law must be replaced by

$$
p=Z(p, T) n k T
$$

where $Z(p, T)$ is the compressibility factor $(\leq 1)$ for the gas, $n$ the gas number density, $k$ Boltzmann's constant and $T$ the absolute gas temperature. For $\mathrm{SF}_{6}$, the influence of compressibility may be accounted for by replacing $p$ with $n$ [18]. This approach, which is supported by Paschen curve breakdown voltage measurements $[19,20]$, implies the existence of a constant $(E / n)_{\text {lim }}$. However, from a practical point of view $n$ is not a satisfactory parameter. It is more convenient to introduce the compressibility-corrected gas pressure $p_{z}$, defined as

$$
p_{z} \equiv \frac{p}{Z(p, T)}
$$

such that when using (9) and (12) to evaluate $\xi$ we simply replace $p$ with $p_{z}$.

As $Z$ may be expressed as a power series in $p$, the second order expression given in [21] is appropriate for the pressure range of interest, see Appendix. In addition, from the values of $(E / p)_{l i m}$ given in $[15,22]$, we can deduce that for $\mathrm{SF}_{6}$ the constant $\left(E / p_{z}\right)_{l i m}$ is 87.9 $\mathrm{kV}(\mathrm{mm} \mathrm{MPa})^{-1}$ while $M=0.004 \mathrm{MPa} \mathrm{mm} \mathrm{[22].} \mathrm{In}$ relation to the onset measurements, the gas temperatures were $19.6^{\circ} \mathrm{C}$ in all cases [14]. The results of the $\xi$ evaluation are shown in Figure 4, which illustrates the variation of this parameter with $p_{z}$.

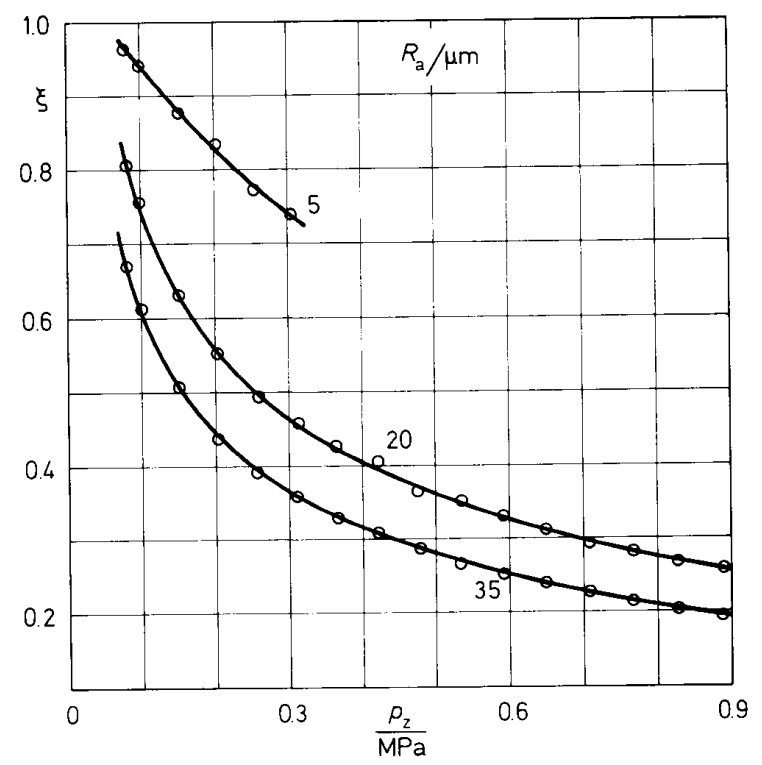

Figure 4.

Variation of the surface roughness factor $\xi$ for $\mathrm{SF}_{6}$ with compressibility-corrected gas pressure $p_{z}$ for different $R_{a}$ values.

\section{REGRESSION ANALYSIS}

On the basis of (13) a polynomial regression analysis of the $\left(\xi, p_{z}\right)$ data was undertaken. As indicated in [23] however, such an analysis might prove to be unsatisfactory with a limited data set. In the present case the $a_{0}$ coefficients obtained exceeded the approximate values of $\xi_{\text {lim }}$ suggested by Figure 4 and, in addition, the remaining coefficients alternated in sign. Consequently, it is preferable to base the regression analysis on an expression of the form

$$
\xi\left(p_{z}\right)=\xi_{\text {lim }}+f\left(p_{z}\right)
$$

with the function $f\left(p_{z}\right) \rightarrow 0$ monotonically as $p_{z} \rightarrow \infty$. For the present $\left(\xi, p_{z}\right)$ data an exponential function was found to be appropriate, with the relationship used in the regression analysis given by

$$
\xi\left(p_{z}\right)=\xi_{\text {lim }}+a \exp \left(b p_{z}\right)
$$

where $a$ and $b$ are the regression coefficients. The values of $m$ derived from this analysis are listed in Table 1.

In performing the analysis, the value of $\xi_{\text {lim }}$ was varied for any set of $\left(\xi, p_{z}\right)$ data until the coefficient of 
Table 1.

Field enhancement factors derived from the $\xi_{\text {lim }}$ evaluation.

\begin{tabular}{ccc}
\hline \hline$R_{a}$ & $m$ & $r^{2}$ \\
$\mu \mathrm{m}$ & & \\
\hline 5 & 2.8 & 0.997 \\
& $(2.1-10.0)$ & \\
20 & 5.6 & 0.998 \\
& $(5.1-6.3)$ & \\
35 & 7.1 & 0.998 \\
& $(6.6-7.8)$ & \\
\hline \hline
\end{tabular}

determination $r^{2}$ displayed a maximum value. The corresponding $m$ value is the principal one listed in Table 1 . As there was no pronounced variation in the $r^{2}$ value around its maximum value, we used this behavior as a measure of the uncertainty in the $\xi_{\text {lim }}$ determination. The associated range in the $m$ values for $r^{2}$ effectively equal to $\left(r^{2}\right)_{m a x}$ is lipted in Table 1 for each $R_{a}$ value. Furthermore, with the 20 and $35 \mu \mathrm{m}$ data the number of $\left(\xi, p_{z}\right)$ values considered was varied to obtain an optimum value of $\left(r^{2}\right)_{\max }$. This was achieved with the $\xi$ measurements in the range $0.25 \leq p \leq 0.8 \mathrm{MPa}$. For $R_{a}=5 \mu \mathrm{m}$ this option was not available as the $U_{o}$ measurements could only be performed up to $0.3 \mathrm{MPa}$. As a consequence, the regression fit in this case was not as good, see Table 1, and thus a greater uncertainty exists in the $\xi_{\text {lim }}$ determination.

\section{DISCUSSION}

A method of determining the field enhancement fac$A$ tor of a rough surface has been developed. The method is based upon the measurement of the voltage associated with discharge onset at the surface under consideration. It is important to note that the method is not dependent on a particular strongly electronegative gas. This flexibility arises owing to the fact that $m$ is a property of the conductor surface alone. Thus any gas in this category could be employed, provided that a meaningful variation in $\xi$ is possible.

To obtain such a $\xi$ variation discharge measurements must be performed at $p>0.1 \mathrm{MPa}$, and as a consequence of the inherent dielectric strength of strongly electronegative gases applied voltages of $>100 \mathrm{kV}$ invariably will be required. When performing the $U_{o}$ measurements at these voltage levels, precautions must be taken to minimize possible changes in surface topography due to sparking, e.g. the discharge energy should be limited. At the higher gas pressures, this requirement can be waived in a coaxial geometry as $U_{o}$ becomes progressively less than the sparking voltage as the pressure increases [17]. This behavior, which is associated with the onset of corona stabilization, is much more pronounced with negative polarity. In addition, Crichton et al. [24] have clearly demonstrated with artificial protrusions that negative polarity should be employed in order to obtain true $U_{o}$ values.

It should be noted that, from the streamer criterion, the discharge onset voltage $U_{o}$ is the minimum voltage level required to initiate either a direct breakdown or a corona discharge [8]. An inherent assumption of this criterion is the existence of a suitably placed initiatory electron, such that the value of the statistical time lag $t_{s}$ is zero. In general $t_{s}>0$, and hence if the applied voltage increases in time, the discharge will occur at a higher voltage level. This voltage is usually referred to as the discharge inception voltage $U_{i}$, with $U_{i} \rightarrow U_{o}$ as $t_{s} \rightarrow 0$. Thus it is only by preselecting the experimental conditions to ensure that $t_{s}$ is minimized, that valid $U_{o}$ measurements can be undertaken.

The current pulses detected at discharge onset are associated in this study with the complete electrode [16], and thus it is impossible to identify discharge development with a particular surface location. Hence the $m$ value derived from the $U_{o}$ measurements is considered to characterize the rough surface as a whole. Spatial resolution could be accomplished by employing an open gap geometry, e.g. a sphere/plane gap. However the major advantage of the coaxial geometry would be lost, namely the large surface area associated with $E_{a}$. This feature promotes the determination of a true onset voltage level, which ensures an $m$ value extremum.

A comparison of Figures 1 and 4 illustrates an inherent limitation in the method. In analytical studies, it is a simple matter to cover several decades of relevant $p$ values. In practice this situation cannot be realized, and in general $U_{o}$ measurements will be restricted to a single decade: $0.1 \leq p \leq 1 \mathrm{MPa}$. This limitation on the experimental pressure range implies that $m$ values derived from the regression analysis will have an inherent uncertainty.

The regression analysis of the $\left(\xi, p_{z}\right)$ data yielded $m$ values of $2.8,5.6$, and 7.1 for the three $R_{a}$ values of 5,20 , and $35 \mu \mathrm{m}$, respectively. As is evident from 
Table 1, the greater the pressure range investigated, the better is the regression fit and the narrower is the range in the possible $m$ values. With reference to the $5 \mu \mathrm{m}$ results, the tabulated upper limit of the $m$ range should be discounted in this case because the true value cannot exceed the $m$ values associated with the 20 and $35 \mu \mathrm{m}$ surfaces. Consequently, the pressure range over which the $5 \mu \mathrm{m}$ surface was examined is clearly inadequate.

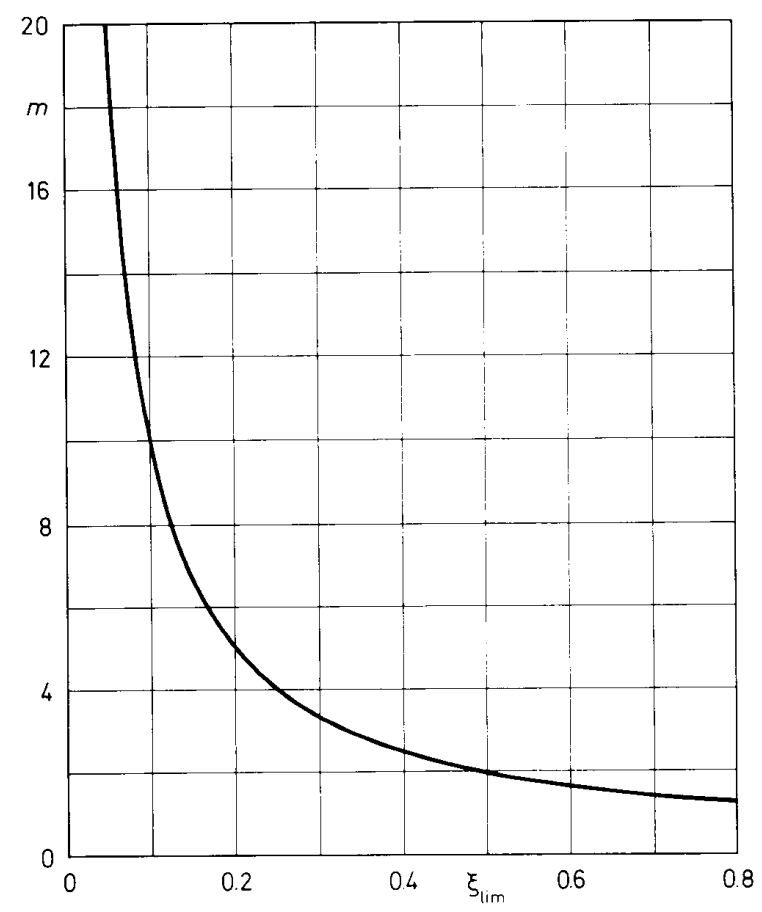

Figure 5.

Variation of the field enhancement factor $m$ with the limiting surface roughness factor $\xi_{\text {lim }}$.

The importance of investigating as large a pressure range as possible even with large $R_{a}$ values may be indicated by considering the nature of the theoretical $\left(\xi_{\text {lim }}, m\right)$ relationship, see $(4)$ and Figure 5. From this diagram it is clear that for a fixed uncertainty on the experimentally derived $\xi_{\text {lim }}$ value the corresponding uncertainty on $m$ is significantly increased at the higher $m$ values. This behavior underlines the necessity of minimizing the uncertainty in the $\xi_{\text {lim }}$ determination for large $R_{a}$ values.

As mentioned previously, the pressure range which could be investigated with the smallest $R_{a}$ value was very restricted owing to an upper limit on the available test voltage; see Figure 3. This situation could be improved by employing a conductor of smaller diameter, i.e. by reducing the value of $\eta$ the corresponding values of $U_{o}$ would be reduced, and thus an extension of the pressure range should be feasible.

In the present study, the rough surfaces which have been analyzed were produced by a turning process which provided a regular ridged surface [15]. As the macroscopic radius of each inner electrode was $11 \mathrm{~mm}$, the associated microscopic geometry was essentially two dimensional; i.e. $\rho_{1} \ll \rho_{2}$. In addition, as the value of the ridge spacing to tip radius of curvature was $>10$, a rough estimate of the $m$ value for these surfaces can be obtained by considering a single isolated ridge exhibiting the same $h / \rho_{1}$ ratio, where $h$ is the ridge height and $\rho_{1}$ its radius of curvature.

For a hemi-elliptical ridge, it can be shown that $m$ is given by

$$
m=1+\sqrt{\frac{h}{\rho_{1}}} \quad\left(\rho_{2}=\infty\right)
$$

In [15] the $R_{a}$ measurements were undertaken using a 3 $\mu \mathrm{m}$ stylus, and we will assume this to be the minimum $\rho_{1}$ value. Consequently for the peak-to-trough values given in [15] for the 5 and $35 \mu \mathrm{m}$ surfaces, we obtain the following estimates for $m: m_{5}=3.2$ and $m_{35}=6.8$. Considering the nature of the approximation, these values are in agreement with the experimentally derived values listed in Table 1 . This agreement confirms that the $m$ values derived from the $U_{o}$ measurements are reliable.

It should be noted that an $R_{a}$ of $35 \mu \mathrm{m}$ represents a very rough surface, and it may be taken as an upper limit for the production processes associated with GIS systems, see $[3,4]$. Caution should be exercised, however, as surfaces with the same $R_{a}$ values can exhibit widely different microscopic geometries [25], and hence generate quite different field enhancement factors. Such a situation with the same $R_{a}$ values can arise following different production processes. 


\section{CONCLUSIONS}

$\mathrm{D}$ ESPITE the geometrical complexity of rough surfaces, it has been possible to develop an indirect method to determine the field enhancement factor $m$ associated with the surface of a practical conductor. This $m$ value characterizes the surface as a whole, and, provided an adequate pressure range is investigated, the $m$ value can be derived with sufficient accuracy for practical purposes.

The implementation of the method is not restricted to a specific strongly electronegative gas and, in addition, analysis of the experimental data is independent of the nature of the microscopic surface roughness.

Finally, it must be emphasized that the $m$ determination is based on continuum theories and consequently the $m$ value should not be related to phenomena on an atomic scale.

\section{ACKNOWLEDGMENT}

$\mathrm{T}$ HE authors wish to thank the Electric Power Research Institute for supporting this work through project RP2669-1.

\section{APPENDIX}

$\mathrm{F}$ or completeness, we include the expression for the compressibility factor $Z(p, T)$ for $\mathrm{SF}_{6}$ given by Vibholm and Mollerup [21]:

$$
Z(p, T)=1+A p+B p^{2}
$$

where

$$
A=\frac{15.84}{T}-\frac{1.280 \times 10^{4}}{(T)^{2}}-\frac{1.368 \times 10^{8}}{(T)^{4}}(\mathrm{MPa})^{-1}
$$

and

$$
\begin{aligned}
& B=-\frac{302.2}{(T)^{2}}+\frac{5.274 \times 10^{5}}{(T)^{3}}-\frac{1.639 \times 10^{8}}{(T)^{4}} \\
& +\frac{2.166 \times 10^{9}}{(T)^{5}}-\frac{3.501 \times 10^{12}}{(T)^{6}}-\frac{1.870 \times 10^{16}}{(T)^{8}}
\end{aligned}
$$

$p(\mathrm{MPa})$ and $T(\mathrm{~K})$ are the absolute pressure and temperature of the gas, respectively.

\section{REFERENCES}

[1] A. Pedersen, "The Effect of Surface Roughness on Breakdown in SF 6 ", IEEE Trans. Power Appar. Syst., Vol. 94, pp. 1749-1754, 1975.

[2] A. Pedersen and E. Bregnsbo, "Estimation of Breakdown Voltages in Compressed $\mathrm{SF}_{6}$ ", Internationales Symposium Hochspannungstechnik, Zürich 1975. Schweizerischer Elektrotechnischer Verein, Zürich , pp. 432-436, 1975.

[3] American National Standard, Surface Texture (Surface Roughness, Waviness and Lay), ANSI B46.11978, The American Society of Mechanical Engineers NY, 1978.

[4] I. W. McAllister, "On the Concept of Electrode Surface Roughness with Reference to Discharge Phenomena in Strongly Electronegative Gases", IEEE Trans. Elect. Insul., Vol. 21, pp. 659-662, 1986.

[5] W. Mosch and W. Hauschild, "Möglichkeiten zur Berechnung der Durchschlagspannung schwach inhomogener Anordnungen im $\mathrm{SF}_{6}$ ", Elektrie, Vol. 28, pp. 152-156, 1974.

[6] I. W. McAllister, "A Multiple Protrusion Model for Surface Roughness Effects in Compressed $\mathrm{SF}_{6}$ ", Elektrotechnische Zeitschrift A, Vol. 99, pp. 283$284,1978$.

[7] I. W. McAllister, "The Influence of Electrode Macroscopic Curvature upon Surface Roughness Effects in Compressed $\mathrm{SF}_{6} "$, Archiv für Elektrotechnik, Vol. 62 , pp. 43-49, 1980.

[8] A. Pedersen, I. W. McAllister, G. C. Crichton and S. Vibholm, "Formulation of the Streamer Breakdown Criterion and its Application to Strongly Electronegative Gases and Gas Mixtures", Archiv für Elektrotechnik, Vol. 67, pp. 395-402, 1984.

[9] J. Berril, J. M. Christensen and I. W. McAllister, "Measurement of the Figure of Merit $M$ for Several Perfluorocarbon Gases", L. G. Christophorou and D. W. Bouldin (eds.), in Gaseous Dielectrics V, Pergamon Press, NY pp. 304-310, 1987.

[10] J. Berril, J. M. Christensen and I. W. McAllister, "Measurement of the Figure of Merit $M$ for Mixtures of Dichlorofluoromethane and Nitrogen", Proc. 18th International Conference on Phenomena in Ionized Gases, Swansea 1987, Adam Hilger Bristol, pp. 672-673, 1987. 
[11] J. Berril, J. M. Christensen and I. W. McAllister, "Measurement of the Figure of Merit $M$ for $\mathrm{CBrClF}_{2}, \mathrm{CBr}_{2} \mathrm{~F}_{2}, \mathrm{CBrF}_{3}$ and $\mathrm{CClF}_{3}$ ", Fifth International Symposium on High Voltage Engineering, Braunschweig, Vol. 1, paper No. 15.16, 1987.

[12] Y. Qiu and Y. F. Liu, "A New Approach to Measurement of the Figure-of-Merit for Strongly Electronegative Gases and Gas Mixtures", IEEE Trans. Elect. Insul., Vol. 22, pp. 831-834, 1987.

[13] J. H. Dymond and E. B. Smith, The Virial Coefficients of Pure Gases and Mixtures: A Critical Compilation, Oxford U. P. Oxford 1980.

[14] G. C. Crichton and S. Vibholm, "Discharge Onset Voltage Prediction for a Gas-Insulated System via the Figure-of-Merit Concept", IEEE Trans. Elect. Insul., Vol. 22, pp. 819-823, 1987.

[15] G. C. Crichton, J. Drimal, I. W. McAllister and S. Vibholm, "Discharge Onset Prediction in 1,2$\mathrm{C}_{2} \mathrm{Cl}_{2} \mathrm{~F}_{4} / \mathrm{SF}_{6}$ Mixtures via the Figure-of-Merit Concept", IX International Conference on Gas Discharges and Their Applications, Venice, pp. 307$310,1988$.

[16] G. C. Crichton and A. Pedersen, "Anomalous Surface Roughness Effects in $\mathrm{SF}_{6}$ ", Sixth International Conference on Gas Discharges and Their Applications, Edinburgh 1980. IEE Conference Publication No. 189, part 1, pp. 210-212, 1980.

[17] S. Vibholm and G. C. Crichton, "On the Optimization of Pressure and Surface Roughness Parameters in Compressed $\mathrm{SF}_{6}$ ", Seventh International Conference on Gas Discharges and Their Applications, London 1982. Peter Peregrinus Ltd. London, pp. 259-261, 1982.

[18] B. H. Crichton and D. J. Tedford, "The Application of Low-Pressure Experimental Data to the Calculation of Electrical Discharge Thresholds in Compressed Gases", J. Phys. D: Appl. Phys., Vol. 9, pp. 1079-1083, 1976.
[19] R. Baumgartner, "Erreichbare Durchschlagfeldstärken in $\mathrm{SF}_{6}$ bei hohem Druck", Elektrotechnische Zeitschrift A, Vol. 98, pp. 369-371, 1977.

[20] I. C. Somerville and D. J. Tedford, "Electrical Breakdown in Compressed $\mathrm{SF}_{6}$ between Highly Polished Electrodes", L. G. Christophorou, ed., Gaseous Dielectrics II, Pergamon Press, NY pp. 100-107, 1980.

[21] S. Vibholm and J. Mollerup, "On the Compressibility Factor for $\mathrm{SF}_{6}$ ", Sixth International Conference on Gas Discharges and Their Applications, Edinburgh 1980. IEE Conference Publication No.189, part 1, pp. 278-280, 1980.

[22] J. Berril, J. M. Christensen and A. Pedersen, "Measurement of the Figure of Merit Related to the Effect of Electrode Surface Defects on Breakdown for Strongly Electronegative Gases or Gas Mixtures", Seventh International Conference on Gas Discharges and Their Applications, London 1982. Peter Peregrinus Ltd. London, pp. 266-268, 1982.

[23] J. H. Mathews, Numerical Methods for Computer Science, Engineering, and Mathematics, PrenticeHall International London, Chap. 5, 1987.

[24] B. H. Crichton, D. I. Lee and D. J. Tedford, "Prebreakdown in Compressed $\mathrm{SF}_{6}$ and $\mathrm{SF}_{6} / \mathrm{N}_{2}$ Mixtures in Projection Perturbed Uniform Fields", 4th International Conference on Gas Discharges, Swansea 1976. IEE Conference Publication No. 143, pp. 199-202, 1976.

[25] T. R. Thomas (ed.), Rough Surfaces, Longman London 1982.

Manuscript was received on 13 Feb 1989 